\title{
Neologismen im Gegenwartsdeutschen - Probleme in Theorie und Praxis
}

\author{
Neologisms in Contemporary German - Problems in Theory and Practice
}

\begin{abstract}
ZUSAMMENFASSUNG
Dieser Artikel beschreibt zunächst die aktuelle Forschung zu neuen Wörtern. Dann werden einige Probleme angesprochen. Die Neologismenforschung des Deutschen zeichnet sich durch lexikographische Dominanz und wenig linguistische Auseinandersetzung aus. Außerdem basieren lexikographische Projekte auf Zeitungs- und Zeitschriftenkorpora und setzen diese mit der deutschen Sprache gleich. Die lexikographische Forschung deckt jedoch nur einen Teil der Fragen zum Thema neue Wörter ab. Gleichzeitig bestimmen zugrunde liegenden Theorie, Fragen und Zielen die Methoden und Vorgehensweisen mit, sodass sich sprachwissenschaftliche und lexikographische Ansichten und Verfahren unterscheiden. Die Lexikologie will verstehen und nicht nur beschreiben und berücksichtigt zudem auch Sprachvarietäten. Dies führt zu einer größeren Vielfalt von Neologismen, als es Wörterbücher vermuten lassen.

Schlüsselwörter: Deutsch, Neologismus, Varietäten, Lexikologie, Lexikographie
\end{abstract}

\section{ABSTRACT}

This article first traces current research on new words. Then some problems are addressed. Neologism research on the German language is characterized by lexicographic dominance and inadequate linguistic analysis. Furthermore, lexicographic projects use newspapers and magazines as a data basis and equate this with the German language. Lexicographic research only covers a subset of the questions on the topic of new words, and methods are influenced by underlying theory, questions and goals, so that linguistic and lexicographic opinions and procedures differ. The aim of linguistic work is to understand and not just to describe. Additionally, language varieties are considered. This leads to greater diversity of neologisms than dictionaries suggest. Keywords: German, neology, varieties, lexicology, lexicography

\section{Einleitung}

Die Neologismenforschung der deutsche Sprache besteht zu einem weiten Teil aus lexikographischen Arbeiten und nur wenig linguistischer Auseinandersetzung. Damit fehlen auch Grundsatzdiskussionen zu Theorie des Neologismus und Methodik der Datenerfassung und -bearbeitung. Universitätsexterne Institutionen wie das Leibniz-Institut für Deutsche Sprache (im Folgenden kurz: IDS) in Mannheim behandeln das Thema ,neue Wörter“" aus praktisch-lexikographischer Sicht. Ziel ist

Hilke Elsen, Institut für Deutsche Philologie, Ludwig-Maximilians-Universität München, Schellingstr. 3/RG, 80799 München, hilkee@Irz.uni-muenchen.de, https://orcid.org/0000-0003-4109-6363 
die Erstellung eines Wörterbuchs für Neologismen der deutschen Allgemeinsprache. Als Datengrundlage dienen Zeitungen, Zeitschriften und Wörterbücher. Entsprechend beziehen sich die Fragestellungen sehr eng auf Deskription und aufWörterbuchpraxis. Linguistische Untersuchungen zu neuen deutschen Wörtern sind hingegen begrenzt, denn politisch motivierte Umstrukturierungen und Kürzungen schränken die Forschung an Universitäten ein. Das führt zum einen dazu, dass lexikographische Resultate speziell des IDS verallgemeinert werden, obwohl selbst andere Projekte wie Duden oder Quasthoff Ergebnisse und Schlussfolgerungen daraus relativieren könnten. Zum anderen basieren Wörterbucheinträge auf Daten aus Zeitungen und Zeitschriften. Infolgedessen werden neue Wörter des Deutschen mit den Ergebnissen der IDS-bezogenen Wörterbuchforschung gleichgesetzt und die Allgemeinsprache mit Zeitungsdeutsch. Wie ist nun der aktuelle Stand der deutschen Neologismusforschung? Dieser Frage geht der Artikel im Folgenden nach (vgl. auch Elsen, im Druck).

\section{Begriff}

Neologismen sind allgemein neue Wörter, im weiteren Sinne zählen dazu auch Okkasionalismen (Einmal- und Gelegenheitsbildungen). Neue Wörter entstehen zumeist durch Wortbildung (Kinderunivorlesung, Supermond), aber auch durch Fremdwortübernahme (Brexit, Wrap), Bedeutungsveränderung (divers für das dritte Geschlecht, Geisterflug [Flug ohne Passagiere]) oder, selten, Kunstwortbildung (Froop, Zalando).

\section{Lexikographie}

Eines der ambitioniertesten lexikographischen Projekte zur deutschen Sprache wird am IDS in Mannheim durchgeführt. Bisher sind zwei Wörterbücher zu Neologismen entstanden, die die letzten zehn Jahre des letzten Jahrhunderts und die ersten zehn des neuen Jahrhunderts umfassen (Herberg, Kinne, \& Steffens, 2004; Steffens \& al-Wadi, 2015, ausführlich vgl. Online-WortschatzInformationssystem Deutsch, im Folgenden kurz: OWID). Ziel des Projekts ist es einerseits, Informationen zu neuen Wörtern, etwa zu Aussprache, Schreibung, Grammatik, Gebrauch, Entstehung, Geschichte zu dokumentieren und einem breiten Publikum, online und als Printmedium, zugänglich zu machen, andererseits auch Daten für die Forschung zu Verfügung zu stellen. Die Arbeit ist daher praktisch-deskriptiv ausgelegt.

Die terminologischen Überlegungen orientieren sich dabei zum einen vorrangig an der Praktikabilität im Rahmen neologismenlexikographischer Arbeit, sie sind aber zum anderen auch bemüht um eine angemessene Balance zwischen fachspezifischer Exaktheit und einer Verständlichkeit nach außen (Kinne, 1996, S. 342).

Damit liegt der Fokus auf Wörtern, die für die Benutzer*innen wahrscheinlich auf irgendeiner Ebene schwierig bzw. interessant sind, vorzugsweise Fremdwörter 
und gerade nicht Komposita, wenn sie keine Verständnisschwierigkeiten bieten. Das führt zu einem der wichtigsten Probleme überhaupt, nämlich der verzerrten Repräsentativität der Daten. Zum anderen sollen die Neologismen zur deutschen Allgemein- bzw. Standardsprache gehören. Entsprechend lautet die Definition:

Ein Neologismus ist eine neue lexikalische Einheit bzw. eine neue Bedeutung einer etablierten lexikalischen Einheit, die in einem bestimmten Abschnitt der Sprachentwicklung in einer Kommunikationsgemeinschaft aufkommt, sich ausbreitet und als sprachliche Norm allgemein akzeptiert wird (Steffens \& al-Wadi, 2015, S. xii).

Seltene Formen, Fach-, Sondersprachen, Regionalismen und Okkasionalismen werden ausgeschlossen, ebenso Namen. Die Verbreitungsgrade werden über Vorkommenshäufigkeiten in den Daten bestimmt. Für die Bearbeitung ergeben sich, darüber sind sich die Mitarbeiter*innen durchaus bewusst, subjektive Entscheidungen und breite Grauzonen.

Während zu Beginn der Arbeit hin und wieder darauf hingewiesen wurde, dass es um eine Auswahl gehen sollte und das Ziel des ersten IDS-Projekts „die lexikografische Beschreibung und Dokumentation von ca. 800 ausgewählten, kommunikativ relevanten Neologismen der 90er Jahre" (Herberg, 2004, S. 332) war, wird der Gegenstand des Wörterbuchs später meist allgemeiner bestimmt als „lexikalische Einheiten bzw. Bedeutungen etablierter lexikalischer Einheiten, die in den Nullerjahren in der deutschen Allgemeinsprache aufgekommen sind [... $]^{\text {“ }}$ (Steffens \& al-Wadi, 2015, S. xii). Dies suggeriert eine gewisse Vollständigkeit.

Die beiden Neologismenwörterbücher führen 654 (Holz, 2009, S. 47) bzw. 570 (Steffens \& al-Wadi, 2015, S. XIII) Stichwörter aus jeweils einem Jahrzehnt auf. Online sind es etwas mehr. Für die Zeit von 1991 bis heute, also für knapp 30 Jahre, sind es etwa 2100 Stichwörter (OWID, 03.11.2020). Im Vergleich dazu nimmt der Rechtschreib-Duden etwa 3000 neue Stichwörter in der 28. Ausgabe auf (gegenüber der 27. von 2017, diese wiederum 5000 gegenüber der 26. von 2013), z.B. Elektroscooter, helikoptern oder Flugscham, ohne allerdings die Kriterien genauer zu bestimmen. Diesbezüglich sind die IDS-Mitarbeiter*innen sehr informativ. Jedoch berücksichtigt die Dudenredaktion durchaus Fachsprachliches, regionale und Gruppenvarietäten sowie Namen. Quellen sind hauptsächlich Zeitungen, aber auch Romane und Sachtexte (Duden, 2017, S. 7-8). Ob die stark unterschiedlichen Ergebnisse auf der anderen Datenbasis oder anderen Erhebungskriterien beruhen, etwa, dass im Duden immer auch Wortgut nachgetragen werden muss, lässt sich somit nicht sagen.

Quasthoff (2007) sammelte für sein Neologismenwörterbuch 2284 neue Wörter zwischen 2000 und 2006, also auch wieder sehrviel mehr als das IDS. Sie stammen aus demLeipzigerKorpusDeutscher Wortschatz (http://www.wortschatz.uni-leipzig.de), wieder basierend auf Zeitungen. Als Kandidaten kamen sie in Betracht, wenn ihr 
Vorkommen ab $2000 \mathrm{im}$ Vergleich zu den fünf Jahren davor stark zugenommen hat und ,[d]ie Anzahl der Nennungen im Jahr der maximalen Häufigkeit [...] eine Mindestanzahl" (Quasthoff, 2007, S. 10) übersteigt. Diese Zahl wurde nicht genannt. Hier waren Eigennamen, aber auch komplexe Wörter mit Namen wieder ausgeschlossen (Irak-Abenteuer) sowie Titel, weibliche Formen und begrenzt auftretende Regionalismen. Allerdings berücksichtigt der Autor explizit auch transparente Komposita (Vogelgrippealarm). Beispiele für seltene Wörter sind Anreicherungsaktivität (49 mal belegt), Vogelgrippegebiet (60), Visadebatte (66), für deutlich häufigere Praxisgebühr (6092), Billigflieger (7490) oder Atomstreit (8256). Im Endeffekt ist auch diese Sammlung nicht mit den IDS-Daten vergleichbar. Da aber alle drei Projekte explizit Wörter der Allgemeinsprache berücksichtigen, wäre ein kritischer Abgleich, zumindest jedoch ein relativierender Hinweis, wünschenswert, vor allem, da sich die Ergebnisse so stark unterscheiden. Da es in der Lexikographie zu erheblichen Normunsicherheit kommt (Holz, 2009, S. 5), bedeutet die Repräsentativität der Sammlungen an sich das erste Problem.

Ohne ein Wörterbuch zusammenstellen zu wollen sammelt Lemnitzer für Die Wortwarte neue Wörter auf täglicher Basis aus Zeitungs- und Zeitschriftentexten. Seine Intention ist es, neue Wörter des heutigen Deutsch zu dokumentieren. Etwa zwölf neue Wörter kommen täglich zur Liste hinzu, wobei er die interessantesten auswählt (Lemnitzer, 2007, S. 78). Okkasionalismen sind dieses Mal dabei, aber aufgrund der technischen Bedingungen keine Neubedeutungen. So kam er für die Jahre zwischen 2000 und 2007 auf 25.000 neue Wörter (S. 78). Für den 4. Februar 2020 gab es sechs Einträge, beispielsweise Assistenztool, Fake-Stau, Infodemie (Information + Epidemie zum Coronavirus) oder Ökomodernismus.

Neuerdings bietet auch die Redaktion des Digitalen Wörterbuchs der Deutschen Sprache (DWDS) eine Zusammenstellung neuer Wörter an. Hier zeigt der Vergleich mit dem Online-Wortschatz-Informationssystem Deutsch (OWID) die Unterschiede. Beide stellen speziell die neuen Wörter zur Coronapandemie (OWID) bzw. zur COVID-19-Pandemie (DWDS) zusammen, die über Wortbildung oder veränderte Bedeutung entstanden sind oder aus einer anderen Sprache bzw. aus einer Fachsprache stammen und nun allgemeinsprachlich verwendet werden. Für den 13. Mai 2020 bestand erstere aus 140, letztere aus 170 Belegen. Unter A und B fanden sich bei OWID Abstrichzentrum, Alltagsmaske, Anderthalbmetergesellschaft, Arbeit auf Distanz, Balkonkonzert, Balkonmusik, Behelfsmaske, Behelfsmundschutz, im DWDS Absonderung, Aerosol, Alltagsmaske, Ansteckungsgefahr, Antikörpertest, asymptomatisch, Atemmaske, Atemschutzmaske, Ausgangsbeschränkung, Ausgangssperre, Ausgangsverbot, Ausgehverbot, Ausstiegsrate, Ausstiegsszenario, Balkonien, Basisreproduktionszahl, Beatmungsgerät, Beatmungsmaschine, Beatmungsmaske, Beatmungsplatz, Beatmungstherapie, Behelfsmaske, Beitragsrückerstattung, Besuchsverbot, Bewegungsprofil, Blutserum. Es gibt nicht nur ganze zwei 
Übereinstimmungen (Alltagsmaske, Behelfsmaske), sondern auch wesentlich mehr Komposita im DWDS. Auch dies sollte die IDS-Daten stark relativieren.

All diese Projekte verwenden unterschiedliche Kriterien für die Aufnahme der Stichwörter, was den Vergleich etwas beschränkt. Sie zeigen dennoch die Bandbreite der Möglichkeiten auf, zu den neuen Wörtern des Deutschen zu gelangen und auch, wie weit entfernt wir von einer repräsentativen, geschweige denn authentischen Zusammenstellung letztendlich sind.

Zudem wollen diese Projekte die Allgemeinsprache bearbeiten, indem sie als Quellen Zeitungen und Zeitschriften ${ }^{1}$ verwenden. Dieses Register wird teilweise als stellvertretend für die Allgemeinsprache angenommen, dies dürfte das zweite wesentliche Problem der lexikographischen Arbeit überhaupt darstellen. Das dritte Problem sind die unterschiedlichen Kriterien, etwa, Namen und transparente Komposita auszuschließen, oder die Grenze zwischen Okkasionalismen und Neologismen über eine bestimmte Häufigkeitszahl zu setzen. Inwiefern tatsächlich DIE neuen Wörter DES Deutschen zusammengestellt werden lässt sich bezweifeln. Dies ist gerade deswegen kritisch, weil die IDS-Neologismen mit denen des Standarddeutschen gleichgesetzt werden. "Neologisms in Standard German which came up in the Nineties have been described comprehensively in a dictionary of neologisms" (Steffens, 2017, S. 275). Als Informationsgewinn für „einige“ neue Wörter aus der Zeitungssprache leistet das Projektergebnis sicher einen sehr erfolgreichen Beitrag, aber nicht für Neologismen der deutschen Sprache, wie der impressionistische Vergleich mit der DWDS-Sammlung andeutet.

\section{Lexikologie}

Linguistische Arbeiten widmen sich anderen Fragestellungen, Themen und Zielen. Aufgrund der schwierigen Forschungssituation in den deutschsprachigen Ländern finden wir linguistische Studien, die sich mit Einzelwörtern oder größeren Daten beschäftigen, nicht sehr oft. Die wenigen Arbeiten der letzten dreißig Jahre unterscheiden sich stark in der Wahl der Methode, Vorgehensweise und theoretischen Grundlage. Den meisten Erhebungen liegt die Zeitungssprache zugrunde (Bizukojć, 2011²; Heyne \& Vollmer, 2016; Holz, 2009; Jesenšek, 1998; Matussek, 1994). Peschel (2002) untersuchte sowohl Zeitungstexte als auch Prosa.

Während Wörterbucheinträge deskriptiv orientiert sind und Nutzerbedürfnisse im Vordergrund stehen, geht es Linguist*innen neben dem Erfassen und Beschreiben viel mehr um Funktionen. Das Zusammenstellen der Daten, eine zentrale lexikographische Aufgabe, bildet somit lediglich eine erste, notwendige Etappe in der linguistischen Forschung. Hier müssen dann aber auch tatsächlich

1 Hingegen wertet der Duden auch Romane und Sachtexte, das DWDS auch Belletristik, Gebrauchsliteratur, Wissenschaft sowie gesprochenen Sprache aus.

2 Korpus: Newsletters in Zeitungen und Zeitschriften. 
alle neuen Wörter Berücksichtigung finden, ohne dass eine Redaktion eine Vorauswahl trifft. Das führt zu einer anderen Auffassung von Neologismus, der auch seltene neue Wörter berücksichtigt. Der Begriff Neologismus bezieht sich

auf neue Fremdwörter, Schöpfungen und auf Wortbildungen und Wortgruppenlexeme, die in Form oder Bedeutung oder beidem neu sind, das heißt, sie sind noch nicht in den aktuellen Wörterbüchern der Standardsprache verzeichnet. Orthographische Varianten werden ausgeschlossen (Elsen, 2011b, S. 22).

Ausschlaggebend ist hier vor allem die Tatsache, dass anfangs nicht entscheidbar ist, ob ein neues Wort selten bleibt und wieder verschwindet (Okkasionalismus) oder erst zu einem - per definitionem - häufigeren Neologismus wird.

Gründe für das Erschaffen und Verwenden von Neologismen sind vielfältig. So unterziehen Heyne und Vollmer (2016) in ihrer Untersuchung die neuen Wörter aus zwei Zeitungstypen, Bild und Süddeutsche Zeitung, einer quantitativen und qualitativen Analyse der Formen und Funktionen, wobei die Persuasion im Zentrum der Arbeit steht. Als Ergebnis können sie festhalten, dass sich informations- und meinungsbetonte Texte stark mischen (vgl. auch Elsen \& Dzikowicz, 2005) - ein besorgniserregendes Ergebnis, wenn die Informationsvermittlung eigentlich ein bedeutendes Ziel der Presse sein sollte. Dies wirft auch die Frage auf, inwiefern hier die neuen Wörter so stark funktional bedingt sind, dass das ihren Wert, die Allgemeinsprache zu repräsentieren, einschränkt oder gar in Frage stellt. Außerdem ist zu prüfen, ob Wörter überhaupt rein deskriptiv und ohne Hinzuziehung von Textumgebung und Sprecher*innen und Hörer*innen betrachtet werden können.

Jugend- und Werbesprache bilden gerade nicht gewöhnliche Wörter, denn sie wollen Aufmerksamkeit erregen, den Wert der Produkte verbessern, überraschen, provozieren (Androutsopoulos, 2005; Elsen, 2011b; Faulhaber \& Elsen, 2016; Krieg-Holz, 2005; Tanner, 1993). Entsprechend sind viele Bildungen auf die eine oder andere Weise auffällig. Dies führt in der Jugendsprache zu eher wenig Komposita (Schwachstrom-Akademie ,Sonderschule'), dafür zu mehr Ableitungen (Flexibelchen) und Bedeutungsveränderungen (Folterkammer [Turnhalle]) - und lediglich 6,96 \% Fremdwörtern (skaten) (Elsen, 2011b). Im Gegensatz dazu sollen neue Wörter in Packungsbeilagen von Medikamenten patientenfreundlich, verständlich und transparent sein. Wenig überraschend führt Kan (2002) sehr viele Determinativkomposita auf (Mischinfusion, Kurzinfusion). Neue Tags sollen helfen, Informationen zu erschließen, sie entstehen kreativ und gleichzeitig praktisch. Sie müssen nicht verständlich sein oder sich nach Wortbildungsregeln richten. Aber sie sollen bestimmte Informationsaspekte in einer Wortform verbinden (vgl. Statecharts, Mythesis, Folkrank; Niemann \& Müller, 2010, S. 82). Moulin (2018) konzentriert sich auf die spielerischen neue Wörter (Humanitätsapostel, Räuberzivil). Als Datengrundlage dienen 
lexikographische Sammlungen, bei denen wieder die Entscheidungen der Wörterbuchmacher*innen filternde Wirkung haben. Bei dieser Gelegenheit macht die Autorin auch auf unterschiedliche Aufnahmen und Behandlungen im Dudenund im IDS-online-Wörterbuch aufmerksam.

Werden der Text als Datengrundlage mit herangezogen und alle neuen Lexeme betrachtet, so zeigt es sich, dass neue Wörter Texttyp und Genre anzeigen können (Elsen, 2007a, 2008b, S. 18; Siebold, 2000; Schüler, 2016). Neben der rein referierenden Funktion übernehmen Neologismen nämlich auch textuelle und stilistische Aufgaben, sie helfen dabei, einen Text zu konstituieren und die thematische Progression zu sichern. Sie stellen Konnektivität sicher, helfen, Redundanz $\mathrm{zu}$ vermeiden, verbinden alte und neue Informationen und konzentrieren komplexe Gedankengänge in einem Wort. Sie verweisen, bewerten und stützen den roten Faden. Schließlich haben sie auch ästhetische und unterhaltende Eigenschaften und helfen, ein stilistisches Profil zu bilden (Elsen, 2011b; Peschel, 2002; Siebold, 2000). In Texten der Science Fiction etwa zeugen sie vom kreativen Potential der Autor*innen (Siebold, 2000) und versprachlichen die Fremdheit anhand lautsymbolischer Effekte (Elsen, 2007a, 2007b, 2008a, 2008b, 2016, 2018). In der Kinderliteratur wiederum kommt es auch zu didaktischen Effekten, wenn neue Wörter spielerisch den Kindern helfen, grammatische Regeln zu entdecken und gleichzeitig zu unterhalten (Elsen, 2011a, 2011b, 2013). Auch die Bildung von Kunstwörtern geschieht keineswegs willkürlich, sondern vielmehr systematisch (Elsen, 2007a, 2007b, 2008a, 2008b, 2018), zur Bedeutung von Neologismen für das Studium des Spracherwerbs vgl. schließlich Elsen und Schlipphak (2015).

Andere Forschungen beschäftigen sich mit dem Problem der Übersetzbarkeit von Neologismen (Augustyn, 2010; Gierden Vega \& Hofmann, 2008; Schüler, 2016) oder suchen nach sozialen Veränderungen, die sich in neuen Wörtern widerspiegeln. Die Übernahme des modernen American Way of Life beispielsweise lässt sich auch beim Thema Essen beobachten (Instant Meal, Fast Food, InstantProdukt) (Tanner, 1993, S. 273). Die verschiedenen Fragen und Quellen für Neologismen fügen sich zu einem komplexen Bild zusammen.

\section{Zeitungssprache}

Ein Thema, das bisher kaum verfolgt wurde, ist konkret Vorkommen, Struktur und Funktionen neuer Wörter mit den Varietäten in Zusammenhang zu bringen (vgl. aber Elsen, 2011b). Da sie verschiedene Aufgaben erfüllen müssen, sollten sich auch Unterschiede bei den Neologismen ergeben. Damit erweist sich aber die Zeitungssprache, die den lexikographischen Erhebungen als Quelle für das Deutsche an sich dient, als alleinige Datengrundlage als problematisch.

Fragen, die bei der Gleichsetzung von Zeitung und Standard gar nicht erst gestellt werden, sind, inwiefern Zeitungssprache eine eigene Textsorte ist, 
die möglicherweise einen eigenen Stil aufweist, eigene Aufgaben erfüllt und bestimmte Wortbildungsverfahren bevorzugt, ob sie überhaupt als einheitlich aufgefasst werden kann und inwiefern sie die Allgemeinsprache beeinflusst.

Holz (2009) geht von einer Textsorte aus mit eigenem Sprachstil und eigenem Funktionsprofil - als Instrument für Informationsvermittlung und damit Gebrauchssprache beeinflusst sie die Gegenwartssprache, kann aber auch stilistische Merkmale zeigen. Dadurch weist die Zeitungssprache typischerweise Nominalstil auf, was mit vielen Komposita einhergeht. Stilistische Aspekte führen dann wiederum gehäuft zu Okkasionalismen. Holz prüft, ob die Neologismen des ersten IDS-Wörterbuchs auch nach dessen Erscheinen noch in Zeitungstexten auftauchen und findet, dass über ein Drittel verloren gegangen sind und lediglich $12,39 \%$ eindeutig in die Standardsprache übernommen wurden (S. 55). Sie stellt damit den Neologismenstatus (der sich laut IDS vom Okkasionalismenstatus abhebt) infrage.

Diesem Problem ging auch eine Untersuchung im Raum Augsburg/München, Februar 2020, nach (Elsen \& Kodantke, 2021). 323 Versuchspersonen hatten anhand eines Fragebogens zu entscheiden, inwiefern ihnen 25 Lexeme (u.a. Veneer, Tae-Bo, Midijob, Traubenkraut, Aut-idem-Regelung, Heely, Funkchip, Demenzgarten, Schenkkreis) und 1 Phraseologismus (sich zum Löffel machen) aus der zweiten IDS-Veröffentlichung bekannt sind oder nicht und was sie bedeuten (könnten). Es zeigte sich, dass die Wörter zu 76,6 \% nicht gekannt wurden und lediglich 3,8 \% richtig erklärt werden konnten. Besonders oft wurden Ken-Ken (96,3\%), Schwampel (90,4 \%) und Taikonaut (90,4\%) als nicht bekannt markiert. Anders die Rentnerbravo (54,0 \%), die auch zu 23,6 \% richtig erklärt wurde, oder onshore (von 45,9\% nicht gekannt), das zu 7,2 \% richtig erklärt wurde. Dies bestätigt Holz' Ergebnisse und zeigt, dass sich die IDS-Aufnahmekriterien nicht dafür eignen, neue Wörter im Sinne von Neologismen, die am Ende in den Wortschatz eingehen, zusammenzustellen. Denn die meisten der Einträge waren den Versuchspersonen, zumindest in dieser Stichprobe, gar nicht bekannt, entweder, weil sie vergessen wurden oder weil sie auf ein Textgenre beschränkt sind, das nicht jede*r kennt und das damit wiederum nicht repräsentativ ist. Denn ein weiteres Ergebnis der Studie war, dass der Anteil an nicht gekannten Ausdrücken mit häufigerem Zeitunglesen deutlich zurückging. Diejenigen, die nie Zeitung lesen, hatten in $86,9 \%$ der Fälle angegeben, einen Ausdruck nicht zu kennen, wohingegen die nicht-gekannt-Antworten bei denjenigen, die täglich Zeitung lesen, nur 69,6\% aller Antworten ausmachten. Es stellt sich somit die Frage, ob die Datengrundlage nicht ausgeweitet und der Neologismenbegriff nicht revidiert werden sollte, wenn er de facto Wörter umfasst, die nach zehn Jahren nicht allgemein akzeptiert sind.

Ein anderer Vergleich zeigt, wie stark die Ergebnisse zu Bauweisen und Verhältnissen von Lexemen divergieren können. Bei einer Gegenüberstellung der 
Wörterbucheinträge unter $H$ und $T$ des Duden 1996 und des Wahrig 1997 (Kirkness, 2001) sowie des Wahrig 2002 (Elsen, 2011b) ergaben sich keine besonders hohen Werte für Determinativkomposita, allerdings sehr hohe für Lehnwörter (Tab. 1).

Tabelle 1. Vergleich verschiedener Erhebungen nach Wortbildungsart, in \% (Elsen, 2011b, S. 44, 161)

\begin{tabular}{|c|c|c|c|c|c|}
\hline & $\begin{array}{c}\text { Duden } \\
(1996) \\
\text { in Kirkness } \\
(2001)\end{array}$ & $\begin{array}{c}\text { Wahrig } \\
(1997) \\
\text { in Kirkness } \\
(2001)\end{array}$ & $\begin{array}{c}\text { Wahrig } \\
(2002) \\
\text { in Elsen } \\
(2011 \mathrm{~b})\end{array}$ & $\begin{array}{c}\text { Elsen } \\
(2011 \mathrm{~b}) \\
\text { Zeitung }\end{array}$ & $\begin{array}{c}\text { Elsen } \\
(2011 \mathrm{~b}) \\
\text { gesamt }\end{array}$ \\
\hline ges. (absolut) & 40 & 486 & 569 & 509 & $\sim 4600$ \\
\hline Komposition & 42,5 & 34,7 & 18,63 & 80,94 & $17,1-80,94$ \\
\hline Derivation & & 13,5 & 23,2 & 5,11 & $0,62-27,24$ \\
\hline Kürzung & 5 & 2,7 & 1,76 & 1,18 & $0,15-10,69$ \\
\hline Konversion & 7,5 & 1,6 & 1,41 & 5,11 & $0-16,11$ \\
\hline Rückbildung & & 0,2 & 0,35 & 0,59 & $0-1,39$ \\
\hline Kontamination & & 0,2 & & 0,39 & $0-6,73$ \\
\hline Bedeutungsveränder. & & & 0,18 & 2,55 & $0,78-28,03$ \\
\hline Fremdwortübernahme & 45 & 46,3 & 54,48 & 0,98 & $0,37-21,19$ \\
\hline
\end{tabular}

Das IDS-Projekt beziffert den Anteil der Anglizismen bei den Neologismen wiederholt auf $40 \%$, bei Hybridbildungen auf weitere $20 \%$ (u.a. Herberg, 2004, S. 339). In Tabelle 1 stellen die beiden rechten Spalten im Vergleich dazu die Ergebnisse für neue Wörter rein auf die Zeitungssprache bezogen zusammen sowie die Bandbreite für alle Varietäten der Untersuchung (Elsen, 2011b, zuerst 2004). Hier sind die Werte für die Komposita und Lehnwörter diametral entgegengesetzt. Lemnitzer (2007, S. 96) fand ebenfalls weniger Anglizismen, in einem Auswahlkorpus von 100 Neologismen, das sogar eher "langweilige Beispiele“ gar nicht erst aufnimmt, $10 \%$ rein englische Fremdwörter. Das heißt, eigentlich gibt es wesentlich mehr Komposita und damit weniger Fremdwörter. Liegt das daran, dass die deutsche Sprache doch etwas anderes als die Zeitungssprache ist? Die Auffächerung in der letzten Spalte der Tabelle lässt darauf schließen. Aber wir müssen auch die Aufnahmekriterien für Wörterbucheinträge beachten, die ja explizit an Wörterbuchbenutzer*innen ausgerichtet sind - und damit wieder nicht das genaue Bild der deutschen Neologismen wiedergeben, sondern verzerrte Zahlen. Die Verhältnisse im Neologismenwörterbuch spiegeln lexikographische Einschränkungen wider. Denn für die Wörterbuchmacher*innen sind fehlende Transparenz ein wichtiges Kriterium für die Aufnahme - das schließt die meisten Komposita aus - sowie mögliche Unsicherheiten bei Schreibung, Aussprache und Grammatik - das begünstigt wiederum Fremdwörter. Somit reflektieren die Zahlen die lexikographischen Einschränkungen, die sich nach Konsumentenbedürfnissen 
und Möglichkeiten der Durchführbarkeit richten. Aus beiden Gründen sind solche Ergebnisse nicht repräsentativ für deutschsprachige Neologismen (Elsen, 2011b, S. 45).

Dies wird besonders deutlich, wenn die Ergebnisse für verschiedene Varietäten verglichen werden (Tabelle 2):

Tabelle 2. Möglichkeiten der Wortschatzerweiterung verschiedener Varietäten, in \%, Ausschnitt (vgl. Elsen, 2011b, S. 163)

\begin{tabular}{|c|r|r|r|r|r|r|r|}
\hline Korpus & Chemie & Technik & Jugend & Werbung & Zeitung & Literatur & Kinderliter. \\
\cline { 2 - 8 } & \multicolumn{1}{|c|}{505} & 644 & 503 & 571 & 412 & 540 & 568 \\
\hline Wortbildart. & & & & & & & \\
\hline Komposition & 36,83 & 76,23 & 17,3 & 61,65 & 80,74 & 64,31 & 61,56 \\
\hline Wortgruppenlexembildung & 13,07 & 20,96 & 0 & 9,63 & 3,34 & 0,37 & 1,65 \\
\hline Derivation & 8,12 & 0,62 & 27,24 & 4,2 & 5,3 & 12,64 & 13,66 \\
\hline davon Zusammenbild. & 0 & 0 & 0,6 & 0 & 0,76 & 1,12 & 1,2 \\
\hline Präfixoidbildung & 0 & 0 & 5,17 & 0,35 & 0 & 1,3 & 1,95 \\
\hline Suffixoidbildung & 0 & 0 & 1,19 & 0,18 & 0 & 0,19 & 0 \\
\hline Konversion & 0 & 0 & 6,76 & 1,23 & 5,11 & 16,17 & 3,75 \\
\hline Kurzwort & 10,69 & 0,31 & 6,56 & 0,18 & 1,18 & 0,19 & 0,15 \\
\hline Bedeutungsveränderung & 1,39 & 0,78 & 28,03 & 1,4 & 2,55 & 1,49 & 2,1 \\
\hline Fremdwort & 4,36 & 1,09 & 6,96 & 21,19 & 0,98 & 0 & 0,45 \\
\hline Kontamination & 7,13 & 0 & 1,39 & 0 & 0,39 & 0,74 & 4,95 \\
\hline Kunstwortbildung & 17,43 & 0 & 0 & 0 & 0,2 & 0,19 & 7,66 \\
\hline
\end{tabular}

Es zeigt sich, dass jede Varietät ein eigenes Profil aufweist. Im Jugendwortschatz sind besonders viele neue Wörter durch Derivation und Bedeutungsveränderung entstanden, in der Sprache der Literatur durch Konversion, in der Chemiefachsprache durch Kunst- und Kurzwortbildung. Die Zeitungssprache hat die meisten Komposita und weniger als $1 \%$ Fremdwörter bei den neuen Wörtern gegenüber der Werbung mit $21,19 \%$ Fremdwörtern. Nun stimmt es zwar, dass ein Wort in einer deutschsprachigen Zeitung Teil der deutschen Sprache ist. Aber es gibt auch Wörter, die dazu gehören, aber nicht unbedingt (oft genug) in der Zeitung stehen. Die Zeitungssprache als Gebrauchssprache bildet nur einen Ausschnitt aus den Sprachformen des Deutschen ab. Sie gehorcht eigenen Regeln und verfolgt eigene Ziele, die nicht für die gesamte deutsche Sprache gelten. Das ist einer der Gründe, warum verschiedene Erhebungen von Neologismen zu unterschiedlichen Ergebnissen kommen. Deswegen dürfen Zeitungssprache und Standardsprache auch nicht gleichgesetzt werden, wenn es um statistische Werte zu Wortgebrauch, Fremdwortanteilen bzw. Produktivität von Morphemen oder Wortbildungsarten geht. 
Eben zu den Varietäten und unterschiedlichen Texttypen wären weitere Erhebung nötig, die den angedeuteten Unterschieden weiter nachgehen und auch die „Lebigkeit“ einzelner Wörter in Abhängigkeit von Faktoren wie Semantik, Wortbildungsart, Wortart, Text etc. untersuchen. Denn offenbar bilden gerade sie die Quelle für Neologismen „ein sehr charakteristisches Phänomen von Neologismen [ist] ihre gruppensprachige und domänenspezifische Geltung“" (Munske, 1993, p. 493).

Zusammengefasst kann festgestellt werden:

- Die Ergebnisse verschiedener Wörterbuchprojekte unterscheiden sich.

- Die Ergebnisse verschiedener Wörterbuchprojekte und lexikologischer Untersuchungen unterscheiden sich.

- Die unterschiedlichen Ergebnisse sind auch auf verschiedene Definitionsund Aufnahmekriterien zurückzuführen, z.B. keine Namen, nur einige Determinativkomposita, vor allem Fremdwörter aufzunehmen vs. wirklich alle neuen Wörter.

\section{Schluss}

Die Ergebnisse zu verschiedenen Varietäten und Textsorten zeigen ein wenig homogenes Bild der Neologismenlandschaft, das von Unterschieden in Gebrauchssituation und Funktionen geprägt ist, aber auch von unterschiedlichen Untersuchungsaufgaben und Aufnahmekriterien. Hier entweder bewusst differenzierte Ergebnisse oder aber mehr Einheitlichkeit zu gewinnen setzt wesentlich mehr Daten, aber auch Abstimmung zwischen Studien und Definitionen voraus sowie grundsätzlich mehr Diskussion. Von Fortschritten in Methodik und Theorie sind wir daher noch weit entfernt.

\section{References}

Androutsopoulos, J. (2005). „... und jetzt gehe ich chillen“: Jugend- und Szenesprachen als lexikalische Erneuerungsquellen des Standards. In L. Eichinger, \& W. Kallmeyer (Eds.), Standardvarietäten: Wie viel Variation verträgt die deutsche Sprache? (pp. 171-206). Berlin, New York: De Gruyter.

Augustyn, R. (2010). Neologismen in Science-Fiction - eine kontrastive Analyse der Übersetzungsverfahren am Beispiel des Romans „Revenge of the Sith“. Lublin Studies in Modern Languages and Literature, 34, 35-50.

Bizukojć, K. (2011). Neue Nominalkomposita in deutschen Newsletter-Texten. Frankfurt am Main: Peter Lang Verlag.

Die Wortwarte. Lothar Lemnitzer, Berlin-Brandenburgische Akademie der Wissenschaften. Retrieved February 14, 2020, from www.wortwarte.de.

Duden (2013, 2017, 2020). Die deutsche Rechtschreibung (26th., 27th., 28th. ed.). Berlin: Dudenverlag.

Digitales Wörterbuch der Deutschen Sprache. Retrieved February 12; May 13, 2020, from https:// www.dwds.de.

Elsen, H. (2007a). Die Aufgabe der Namen im literarischen Text. Zeitschrift für Literaturwissenschaft und Linguistik, 147, 151-163. 
Elsen, H. (2007b). Die Wortbildung der Eigennamen in fiktionalen Texten. Zeitschrift für Literaturwissenschaft und Linguistik, 148, 184-197.

Elsen, H. (2008a). Die sprachliche Gestaltung phantastischer Szenarien - die Rolle der Namen. Muttersprache, 118(2), 97-106.

Elsen, H. (2008b). Phantastische Namen. Die Namen in Science Fiction und Fantasy zwischen Arbitrarität und Wortbildung. Tübingen: Narr.

Elsen, H. (2011a). Das besondere Funktionsspektrum der Wort(neu)bildung in der phantastischen Kinderliteratur. In H. Elsen, \& S. Michel (Eds.), Wortbildung im Deutschen zwischen Sprachsystem und Sprachgebrauch. Perspektiven - Analysen - Anwendungen (pp. 211-223). Stuttgart: ibidem.

Elsen, H. (2011b). Neologismen. Formen und Funktionen neuer Wörter in verschiedenen Varietäten des Deutschen (2nd ed.). Tübingen: Narr.

Elsen, H. (2013). Wortschatzanalyse. Tübingen, Basel: Francke.

Elsen, H. (2016). Einführung in die Lautsymbolik. Berlin: Erich Schmidt Verlag.

Elsen, H. (2018). Some names are more equal than others. The sound symbolic value of new names. Cahiers de lexicologie et normes, 113(2), 79-94.

Elsen, H. (in press). German neologisms: what's going on, what's going wrong? Neologica 15. Etat de la recherche européenne sur la néologie: problèmes thèoriques et terminologiques coordonné par Vincent BALNAT et Christophe GERARD.

Elsen, H., \& Dzikowicz, E. (2005). Neologismen in der Zeitungssprache. Deutsch als Fremdsprache, 2, 80-85.

Elsen, H., \& Kodantke, A. (2021). Neologismen aus lexikographischer und lexikologischer Sichteine empirische Untersuchung. Manuscript submitted for publication.

Elsen, H., \& Schlipphak, K. (2015). Word-formation in first language acquisition. In P. O. Müller, I. Ohnheiser, S. Olsen, \& F. Rainer (Eds.), Word-Formation III. An International Handbook of the Languages of Europe (pp. 2117-2137). Berlin: De Gruyter.

Faulhaber, M., \& Elsen, H. (2016). Neologismen in der Kosmetikwerbung. Muttersprache, 3, 193-207.

Gierden Vega, C., \& Hofmann, D. (2008). Wortbildung und Ad-hoc-Komposita: Typen, Implikationen und ihre möglichen Übersetzungen ins Spanische. In L. Eichinger, M. Meliss, \& M. Vázquez (Eds.), Wortbildung heute. Tendenzen und Kontraste in der deutschen Gegenwartssprache (pp. 195-211). Tübingen: Narr.

Herberg, D. (2004). Das Projekt „Neologismen der 90er Jahre des 20. Jahrhunderts“. In J. Scharnhorst (Ed.), Sprachkultur und Lexikographie. Von der Forschung zur Nutzung von Wörterbüchern (pp. 331-353). Frankfurt am Main: Peter Lang Verlag.

Herberg, D., Kinne, M., \& Steffens, D. (2004). Neuer Wortschatz. Neologismen der 90er Jahre im Deutschen. Berlin, New York: De Gruyter.

Heyne, S., \& Vollmer, B. (2016). Innovation und Persuasion in der Presse. Eine komparative Korpusanalyse zur Form und Funktion von Neologismen. Wiesbaden: Springer.

Holz, L. (2009). Untersuchungen zu Neologismen in der Tagespresse. Grundlagen, Erscheinungsformen und Funktionen. Saarbrücken: VDM.

Jesenšek, V. (1998). Okkasionalismen. Ein Beitrag zur Lexikologie des Deutschen. Maribor: Slavistično društvo.

Kan, E. N. (2002). Kreative Wortschöpfungen der Fachlexik in Packungsbeilagen von Medikamenten. Germanistisches Jahrbuch der GUS. Das Wort, 77-92.

Kinne, M. (1996). Neologismus und Neologismenlexikographie im Deutschen. Deutsche Sprache, $24(4), 327-358$.

Kirkness, A. (2001). Europäismen/Internationalismen im heutigen deutschen Wortschatz. In G. Stickel (Ed.), Neues und Fremdes im deutschen Wortschatz. Aktueller lexikalischer Wandel (pp. 105-130). Berlin, New York: De Gruyter. 
Krieg-Holz, U. (2005). Wortbildungsstrategien in der Werbung. Hamburg: Buske.

Lemnitzer, L. (2007). Von Aldianer bis Zauselquote. Neue deutsche Wörter. Wo sie herkommen und wofür wir sie brauchen. Tübingen: Narr.

Matussek, M. (1994). Wortneubildung im Text. Hamburg: Buske.

Moulin, C. (2018). Ludicity in lexical innovation (II) - German. In S. Arndt-Lappe, A. Braun, C. Moulin, \& E. Winter-Froemel (Eds.), Expanding the Lexicon: Linguistic Innovation, Morphological Productivity, and Ludicity (pp. 261-285). Berlin, New York: De Gruyter.

Munske, H. H. (1993). Wie entstehen Phraseologismen? In K. J. Mattheier, K.-P. Wegera, W. Hoffmann, J. Macha, \& H.-J. Solms (Eds.), Vielfalt des Deutschen. Festschrift für Werner Besch (pp. 481-516). Frankfurt am Main: Peter Lang Verlag.

Niemann, C., \& Müller, S. (2010). Ein Tag sagt mehr als tausend Worte? - Kreatives Potenzial und Neotags in Tagging-Systemen. In J. Bermann, \& P. Danowski (Eds.), Handbuch Bibliothek 2.0 (pp. 63-85). Berlin, New York: De Gruyter.

Online-Wortschatz-Informationssystem Deutsch (OWID). Retrieved February 2; May 13; November 3, 2020, from www.owid.de.

Peschel, C. (2002). Zum Zusammenhang von Wortneubildung und Textskonstitution. Tübingen: Niemeyer.

Quasthoff, U. (2007). Deutsches Neologismenwörterbuch. Neue Wörter und Wortbedeutungen in der Gegenwartssprache. Berlin, New York: De Gruyter.

Schüler, A. (2016). Neologismen in der Science Fiction. Eine Untersuchung ihrer Übersetzung vom Englischen ins Deutsche. Frankfurt am Main: Peter Lang Verlag.

Siebold, O. (2000). Wort - Genre - Text. Wortneubildungen in der Science Fiction. Tübingen: Narr.

Steffens D. (2017). Von Pseudoanglizismen und Kurzzeitwörtern. Sprachwissenschaft, 42(3), 275-304.

Steffens, D., \& al-Wadi, D. (2015). Neuer Wortschatz. Neologismen im Deutschen 2001-2010: Vols. 1-2. Mannheim: Institut für Deutsche Sprache.

Tanner, J. (1993). Kulinarische Neologismen in der deutschen Gegenwartssprache. In A. Wierlacher (Ed.), Kulturthema Essen. Ansichten und Problemfeld (pp. 269-277). Berlin: Akademie Verlag. 